\title{
On the local well-posedness of the nonlinear heat equation associated to the fractional Hermite operator in modulation spaces
}

\section{Elena Cordero ${ }^{1}$}

Received: 18 October 2020 / Revised: 8 November 2020 / Accepted: 12 November 2020 / Published online: 11 February 2021

(c) The Author(s) 2021

\section{Abstract}

In this note we consider the nonlinear heat equation associated to the fractional Hermite operator $H^{\beta}=\left(-\Delta+|x|^{2}\right)^{\beta}, 0<\beta \leq 1$. We show the local solvability of the related Cauchy problem in the framework of modulation spaces. The result is obtained by combining tools from microlocal and time-frequency analysis. As a byproduct, we compute the Gabor matrix of pseudodifferential operators with symbols in the Hörmander class $S_{0,0}^{m}, m \in \mathbb{R}$.

Keywords Modulation spaces · Gabor matrix · Fractional Hermite operators · Shubin and Hörmander classes

Mathematics Subject Classification 42B35 $\cdot 35 \mathrm{~K} 05 \cdot 35 \mathrm{~K} 55 \cdot 35 \mathrm{~A} 01$

\section{Introduction and results}

In this note we study the Cauchy problem for the nonlinear heat equation associated to the fractional Hermite operator

$$
\left\{\begin{array}{l}
\partial_{t} u+H^{\beta} u=F(u) \\
u(0, x)=u_{0}(x)
\end{array}\right.
$$

with $t \in[0, T], T>0, x \in \mathbb{R}^{d}, H^{\beta}=\left(-\Delta+|x|^{2}\right)^{\beta}, 0<\beta \leq 1, \Delta=\partial_{x_{1}}^{2}+\ldots \partial_{x_{d}}^{2}$, $d \geq 1$. $F$ is a scalar function on $\mathbb{C}$, with $F(0)=0$. The solution $u(t, x)$ is a complex valued function of $(t, x) \in \mathbb{R} \times \mathbb{R}^{d}$. We will consider the case in which $F$ is a real analytic function with an entire extension.

Elena Cordero

elena.cordero@unito.it

1 Department of Mathematics, University of Torino, Via Carlo Alberto 10, 10123 Torino, Italy 
Well-posedness of the heat equation has been studied by many authors, see e.g. [10,21] and the many contributions by Wong, for instance [31,32], see also [6]. In particular, heat equations associated to fractional Hermite operators were recently studied in [3], for related results see also [5]. Hermite multipliers are considered in [4], see also the textbook [25]. Recently the study of Cauchy problems in modulation spaces have been pursued by many authors, see the pioneering works [1,2]. Many deep results in this framework for nonlinear evolution equations have been obtained by $\mathrm{B}$. Wang et al. in $[27,28]$ and are also available in the textbook [30].

Following the spirit of $[8,12]$, we shall prove the local existence and uniqueness of the solutions in modulation spaces to the Cauchy problem (1). The key arguments come from both microlocal and time-frequency analysis. In fact, we shall rely on the results related to spectral theory of globally elliptic operators developed by Helffer [20] to understand the properties of the fractional Hermite operators $H^{\beta}$. Namely, they are pseudodifferential operators with Weyl symbols $a_{\beta}$ positive globally elliptic and in the Shubin classes $\Gamma_{1}^{2 \beta}$ (see Definition 2.2 and the estimate (13) below).

The spectral decomposition of the Hermite operator $H=-\Delta+|x|^{2}$ is given by $H=\sum_{k=0}^{\infty}(2 k+d) P_{k}$, where $P_{k}$ is the orthogonal projection of $L^{2}\left(\mathbb{R}^{d}\right)$ onto the eigenspace corresponding to the eigenvalue $(2 k+d)$. Namely, the range of the operator $P_{k}$ is the space spanned by the Hermite functions $\Phi_{\alpha}$ in $\mathbb{R}^{d}$, with $\alpha$ multi-index in $\mathbb{N}^{d}$, such that $|\alpha|=k$. The solution to the homogeneous Cauchy problem (1) (i.e., $F=0$ ) can be formally written in terms of the heat semigroup related to $H^{\beta}$

$$
e^{-t H^{\beta}}=\sum_{k=0}^{+\infty} e^{-t(2 k+d)^{\beta}} P_{k}
$$

as $u(t, x)=K_{\beta}(t) u_{0}=e^{-t H^{\beta}} u_{0}(x), \quad t \geq 0, x \in \mathbb{R}^{d}$.

We shall prove that the propagator $K_{\beta}(t)=e^{-t H^{\beta}}$ can be represented as a pseudodifferential operator with Weyl symbol in the Shubin class $\Gamma_{1}^{0}$, with related semi-norms uniformly bounded with respect to the time variable $t \in[0, T]$, for any fixed $T>0$.

After that we shall leave the microlocal techniques to come to time-frequency analysis. We perform a general study concerning the boundedness of Shubin $\tau$ pseudodifferential operators with symbols in the Hörmander classes $S_{0,0}^{m}$ (for $\tau=1 / 2$ we recapture the Weyl case). The outcomes are contained in Theorem 2.4 below (see also the subsequent corollary and remark).

The main tool here is to study the decay of their related Gabor matrix representations, which we shall also control by the semi-norms in $S_{0,0}^{m}$. We think that such result is valuable in and of itself.

We then use the special case of Weyl operators to study (1). The integral version of the problem (1) has the form

$$
u(t, \cdot)=K_{\beta}(t) u_{0}+\mathcal{B} F(u)
$$

where

$$
\mathcal{B}=\int_{0}^{t} K_{\beta}(t-\tau) \cdot d \tau .
$$


To show that the Cauchy Problem (1) has a unique solution, we use a variant of the contraction mapping theorem (see Proposition 3.3 below).

As already mentioned, the function spaces used for our results are weighted modulation spaces $M_{w}^{p, q}, 1 \leq p, q \leq \infty$, introduced by H. Feichtinger in 1983 [14] (then extended to $0<p, q \leq \infty$ in [15]). We refer the reader to Sect. 2 for their definitions and main properties.

The local well-posedness results for modulation spaces read as follows:

Theorem 1.1 Assume $s \geq 0,1 \leq p<\infty, u_{0} \in M_{s}^{p, 1}\left(\mathbb{R}^{d}\right)$ and

$$
F(z)=\sum_{j, k=0}^{\infty} c_{j, k} z^{j} \bar{z}^{k},
$$

an entire real-analytic function on $\mathbb{C}$ with $F(0)=0$. For every $R>0$, there exists $T>0$ such that for every $u_{0}$ in the ball $B_{R}$ of center 0 and radius $R$ in $M_{S}^{p, 1}\left(\mathbb{R}^{d}\right)$ there exists a unique solution $u \in \mathcal{C}^{0}\left([0, T] ; M_{S}^{p, 1}\left(\mathbb{R}^{d}\right)\right)$ to (1). Furthermore, the map $u_{0} \mapsto u$ from $B_{R}$ to $\mathcal{C}^{0}\left([0, T] ; M_{S}^{p, 1}\left(\mathbb{R}^{d}\right)\right)$ is Lipschitz continuous.

For $p=\infty$ the result still holds if we replace $M_{s}^{\infty, 1}\left(\mathbb{R}^{d}\right)$ with the space $\mathcal{M}_{s}^{\infty, 1}\left(\mathbb{R}^{d}\right)$, the closure of the Schwartz class in the $M_{S}^{\infty, 1}$-norm.

We actually do not know whether it is possible to obtain better results concerning the nonlinearity $F(u)=\lambda|u|^{2 k} u, k \in \mathbb{N}$, we refer to the work [5] for a discussion on the topic.

The tools employed follow the pattern of similar Cauchy problems studied for other equations such as the Schrödinger, wave and Klein-Gordon equations [1,2,8,12].

To compare with other results in the literature, we observe that also in [31,32], and in [6] the authors use Wigner distributions and pseudodifferential operators as tools for their main results. In the latter paper the author gives a formula for the oneparameter strongly continuous semigroup $e^{-t H^{\beta}}$ in terms of the Weyl transforms of a $L^{2}$-orthonormal basis made of generalized Hermite eigenfunctions. This is then used to obtain $L^{2}$-estimates for the solution of the related initial value problem with data in $L^{p}$ spaces, $1 \leq p \leq \infty$. Here the approach uses similar ideas of joining microlocal and time-frequency analysis tools, but the spaces employed are different: we use modulation spaces, which are the most common ones in time-frequency analysis.

\section{Function spaces and preliminaries}

We denote by $v$ a continuous, positive, submultiplicative weight function on $\mathbb{R}^{d}$, i.e., $v\left(z_{1}+z_{2}\right) \leq v\left(z_{1}\right) v\left(z_{2}\right)$, for all $z_{1}, z_{2} \in \mathbb{R}^{d}$. We say that $w \in \mathcal{M}_{v}\left(\mathbb{R}^{d}\right)$ if $w$ is a positive, continuous weight function on $\mathbb{R}^{d} v$-moderate: $w\left(z_{1}+z_{2}\right) \leq C v\left(z_{1}\right) w\left(z_{2}\right)$ for all $z_{1}, z_{2} \in \mathbb{R}^{d}$ (or for all $z_{1}, z_{2} \in \mathbb{Z}^{d}$ ). We will mainly work with polynomial weights of the type

$$
v_{s}(z)=\langle z\rangle^{s}=\left(1+|z|^{2}\right)^{s / 2}, \quad s \in \mathbb{R}, \quad z \in \mathbb{R}^{d}\left(\text { or } \mathbb{Z}^{d}\right) .
$$


Observe that, for $s<0, v_{s}$ is $v_{|s|}$-moderate. Moreover, we limit to weights $w$ with at most polynomial growth, that is there exists $C>0, s>0$ such that

$$
w(z) \leq C\langle z\rangle^{s}, \quad z \in \mathbb{R}^{2 d}
$$

We define $\left(w_{1} \otimes w_{2}\right)(x, \xi):=w_{1}(x) w_{2}(\xi)$, for $w_{1}, w_{2}$ weights on $\mathbb{R}^{d}$.

The main features of time-frequency analysis are $T_{x}$ and $M_{\xi}$, the so-called translation and modulation operators, defined by $T_{x} g(y)=g(y-x)$ and $M_{\xi} g(y)=$ $e^{2 \pi i \xi y} g(y)$. Let $g \in \mathcal{S}\left(\mathbb{R}^{d}\right)$ be a non-zero window function in the Schwartz class and consider the short-time Fourier transform (STFT) $V_{g} f$ of a function/tempered distribution $f$ in $\mathcal{S}^{\prime}\left(\mathbb{R}^{d}\right)$ with respect to the the window $g$ :

$$
V_{g} f(x, \xi)=\left\langle f, M_{\xi} T_{x} g\right\rangle=\int e^{-2 \pi i \xi y} f(y) \overline{g(y-x)} d y,
$$

i.e., the Fourier transform $\mathcal{F}$ applied to $f \overline{T_{x} g}$.

For $z=\left(z_{1}, z_{2}\right) \in \mathbb{R}^{2 d}$, we call time-frequency shifts the composition

$$
\pi(z)=M_{z_{2}} T_{z_{1}}
$$

Modulation Spaces. For $1 \leq p, q \leq \infty$ such spaces were introduced by $\mathrm{H}$. Feichtinger in [14] (see also their characterization in [13]), then extended to $0<$ $p, q \leq \infty$ by Y.V. Galperin and S. Samarah in [15].

Definition 2.1 Fix a non-zero window $g \in \mathcal{S}\left(\mathbb{R}^{d}\right)$, a weight $w \in \mathcal{M}_{v}$ and $0<p, q \leq$ $\infty$. The modulation space $M_{w}^{p, q}\left(\mathbb{R}^{d}\right)$ consists of all tempered distributions $f \in \mathcal{S}^{\prime}\left(\mathbb{R}^{d}\right)$ such that the (quasi-)norm

$$
\|f\|_{M_{w}^{p, q}}=\left\|V_{g} f\right\|_{L_{w}^{p, q}}=\left(\int_{\mathbb{R}^{d}}\left(\int_{\mathbb{R}^{d}}\left|V_{g} f(x, \xi)\right|^{p} w(x, \xi)^{p} d x\right)^{\frac{q}{p}} d \xi\right)^{\frac{1}{q}}
$$

(with obvious changes with $p=\infty$ or $q=\infty$ ) is finite.

For $1 \leq p, q \leq \infty$ they are Banach spaces, whose norm does not depend on the window $g$, in the sense that different window functions in $\mathcal{S}\left(\mathbb{R}^{d}\right)$ yield equivalent norms. Moreover, the window class $\mathcal{S}\left(\mathbb{R}^{d}\right)$ can be extended to the modulation space $M_{v}^{1,1}\left(\mathbb{R}^{d}\right)$ (so-called Feichtinger algebra).

For shortness, we write $M_{w}^{p}\left(\mathbb{R}^{d}\right)$ in place of $M_{w}^{p, p}\left(\mathbb{R}^{d}\right), M^{p, q}\left(\mathbb{R}^{d}\right)$ if $w \equiv 1$. Moreover, for $w(x, \xi)=\left(1 \otimes v_{s}\right)(x, \xi)$, we shall simply write, using the standard notation [14],

$$
M_{1 \otimes v_{s}}^{p, q}\left(\mathbb{R}^{d}\right)=M_{s}^{p, q}\left(\mathbb{R}^{d}\right)
$$

In our study, we will apply Minkowski's integral inequality to study the operator $\mathcal{B}$ in (3). Such inequalities do not hold whenever the indices $p<1$ or $q<1$, hence we shall limit ourselves to the cases $1 \leq p, q \leq \infty$. 
We do not know whether the local well-posedness is still valid in the quasi-Banach setting.

Recall that for $1 \leq p, q \leq \infty, w \in \mathcal{M}_{v}$ and $g \in M_{v}^{1}\left(\mathbb{R}^{d}\right)$, the norm $\left\|V_{g} f\right\|_{L_{w}^{p, q}}$ is an equivalent norm for $M_{w}^{p, q}\left(\mathbb{R}^{d}\right)$ [16, Thm. 11.3.7]). In other words, given any $g \in M_{v}^{1}\left(\mathbb{R}^{d}\right)$ and $f \in M_{w}^{p, q}$ we have the norm equivalence

$$
\|f\|_{M_{w}^{p, q}} \asymp\left\|V_{g} f\right\|_{L_{w}^{p, q}}
$$

For this work we will use the inversion formula for the STFT (see [16, Proposition 11.3.2]): assume $g \in M_{v}^{1}\left(\mathbb{R}^{d}\right) \backslash\{0\}, f \in M_{w}^{p, q}\left(\mathbb{R}^{d}\right)$, with $w \in \mathcal{M}_{v}$, then

$$
f=\frac{1}{\|g\|_{2}^{2}} \int_{\mathbb{R}^{2 d}} V_{g} f(z) \pi(z) g d z,
$$

and the equality holds in $M_{w}^{p, q}\left(\mathbb{R}^{d}\right)$.

We also recall their inclusion relations:

$$
M_{w}^{p_{1}, q_{1}} \hookrightarrow M_{w}^{p_{2}, q_{2}}, \quad \text { if } p_{1} \leq p_{2}, q_{1} \leq q_{2}
$$

Other properties and more general definitions of modulation spaces can now be found in textbooks $[9,16]$.

\subsection{Shubin classes and symbols of the operators $H^{\beta}$ and $e^{-t H^{\beta}}$}

Let us first recall the definition of Shubin classes (Shubin [24, Definition 23.1]):

Definition 2.2 Let $m \in \mathbb{R}$. The symbol class $\Gamma_{1}^{m}\left(\mathbb{R}^{2 d}\right)$ consists of all complex functions $a \in C^{\infty}\left(\mathbb{R}^{2 d}\right)$ such that for every $\alpha \in \mathbb{N}^{2 d}$ there exists a constant $C_{\alpha} \geq 0$ with

$$
\left|\partial_{z}^{\alpha} a(z)\right| \leq C_{\alpha}\langle z\rangle^{m-|\alpha|}, \quad z \in \mathbb{R}^{2 d}
$$

It immediately follows from this definition that if $a \in \Gamma_{1}^{m}\left(\mathbb{R}^{2 n}\right)$ and $\alpha \in \mathbb{N}^{2 n}$ then $\partial_{z}^{\alpha} a \in \Gamma_{1}^{m-|\alpha|}\left(\mathbb{R}^{2 n}\right)$.

Obviously $\Gamma_{1}^{m}\left(\mathbb{R}^{2 n}\right)$ is a complex vector space for the usual operations of addition and multiplication by complex numbers, and we have

$$
\Gamma_{1}^{-\infty}\left(\mathbb{R}^{2 d}\right)=\bigcap_{m \in \mathbb{R}} \Gamma_{1}^{m}\left(\mathbb{R}^{2 d}\right)=\mathcal{S}\left(\mathbb{R}^{2 d}\right)
$$

The notion of asymptotic expansion of a symbol $a \in \Gamma_{1}^{m}\left(\mathbb{R}^{2 d}\right)$ (cf. [24], Definition 23.2) reads as follows. 
Definition 2.3 Let $\left(a_{j}\right)_{j}$ be a sequence of symbols $a_{j} \in \Gamma_{1}^{m_{j}}\left(\mathbb{R}^{2 d}\right)$ such that $\lim _{j \rightarrow+\infty} m_{j} \rightarrow-\infty$. Let $a \in C^{\infty}\left(\mathbb{R}^{2 d}\right)$. If for every integer $r \geq 2$ we have

$$
a-\sum_{j=0}^{r-1} a_{j} \in \Gamma_{1}^{\bar{m}_{r}}\left(\mathbb{R}^{2 d}\right)
$$

where $\bar{m}_{r}=\max _{j \geq r} m_{j}$ we will write $a \sim \sum_{j=0}^{\infty} a_{j}$ and call this relation an asymptotic expansion of the symbol $a$.

The interest of the asymptotic expansion comes from the fact that every sequence of symbols $\left(a_{j}\right)_{j}$ with $a_{j} \in \Gamma_{1}^{m_{j}}\left(\mathbb{R}^{2 d}\right)$, the degrees $m_{j}$ being strictly decreasing and such that $m_{j} \rightarrow-\infty$ determines a symbol in some $\Gamma_{1}^{m}\left(\mathbb{R}^{2 d}\right)$, that symbol being unique up to an element of $\mathcal{S}\left(\mathbb{R}^{2 d}\right)$.

The symbol of the Hermite operator (or harmonic oscillator) $H(z)=\left(|x|^{2}+\right.$ $\left.4 \pi^{2}|\xi|^{2}\right)$ obviously belongs to $\Gamma_{1}^{2}\left(\mathbb{R}^{2 d}\right)$.

From [20, Theorem 1.11.1] we infer that the fractional power $H^{\beta}, 0<\beta<1$, can be written as a Weyl pseudodifferential operator having real symbol $a_{\beta}$ in the Shubin class $\Gamma_{1}^{2 \beta}$ and positive globally elliptic. Recall that a symbol $a_{\beta}$ is positive globally elliptic if there exist $C>0$ and $R>0$ such that

$$
a_{\beta}(z) \geq C\langle z\rangle, \quad|z| \geq R .
$$

Thanks to the properties of $H^{\beta}$ above, we can exploit a result by Nicola and Rodino in [22, Theorem 4.5.1] to prove that the operator $e^{-t H^{\beta}}$ is a pseudodifferential operator with Weyl symbol in the Shubin class $\Gamma_{1}^{0}$, with uniform estimates with respect to $t \in[0, T]$, for any fixed $T>0$.

For this purpose, we use the above theorem in the following setting:

$$
\Phi(z)=\Psi(z)=\langle z\rangle, \quad h(z)=\Phi(z)^{-1} \Psi(z)^{-1}=\langle z\rangle^{-2} ;
$$

moreover we choose the parameters $l=N=0$ and $J=1$. If we consider the asymptotic expansion $a_{\beta} \sim \sum_{0}^{\infty} a_{\beta, j}$ as in Definition 2.3, together with the ellipticity condition $a_{\beta, 0}(z) \gtrsim\langle z\rangle^{2 \beta},|z| \geq R$, then Theorem 4.5.1. guarantees that the operator $e^{-t H^{\beta}}$ is a pseudodifferential operator with Weyl symbol $b(t, z)$ satisfying, for every $k \in \mathbb{N}, T>0$, the estimate

$$
\left|b(t, \cdot)-b_{0}(t, \cdot)\right|_{k} \lesssim\langle z\rangle^{-2}, \quad t \in[0, T]
$$

where $b_{0}(t, z)=e^{-t a_{\beta, 0}(z)}$, and the semi-norms $|\cdot|_{k}, k \in \mathbb{N}$, are defined by

$$
|a|_{k}:=\sup _{|\alpha|+|\beta| \leq k}\left|\partial_{\xi}^{\alpha} \partial_{x}^{\beta} a(x, \xi)\right|\langle(x, \xi)\rangle^{|\alpha|+|\beta|} .
$$

Defining the remainder $R_{1}(t, z):=b(t, z)-b_{0}(t, z)$, we infer from (14) that $R_{1}(t, \cdot) \in$ $\Gamma_{1}^{-2}\left(\mathbb{R}^{2 d}\right) \subset \Gamma_{1}^{0}\left(\mathbb{R}^{2 d}\right)$ uniformly w.r.t. $t$ on $[0, T]$. 
Moreover, using the ellipticity condition $a_{\beta, 0}(z) \gtrsim\langle z\rangle$, for $|z| \geq R$, and the property $a_{\beta, 0} \in \Gamma_{1}^{2 \beta}\left(\mathbb{R}^{2 d}\right)$, one easily shows by induction that there exists a constant $C>0$ :

$$
\left|\partial_{z}^{\alpha} b_{0}(t, z)\right| \leq C\langle z\rangle^{-|\alpha|}, \quad \forall t \in[0, T]
$$

that is to say, the symbol $b_{0}(t, z)$ is in the Shubin class $\Gamma_{1}^{0}\left(\mathbb{R}^{2 d}\right)$ with uniform estimates w.r.t. the time variable $t \in[0, T]$. Hence, $b(t, \cdot)=b_{0}(t, \cdot)+R_{1}(t, \cdot) \in \Gamma_{1}^{0}\left(\mathbb{R}^{2 d}\right)$, with uniform estimate w.r.t. $t \in[0, T]$.

For applications of Shubin classes in the framework of Born-Jordan quantization we refer to the work [7].

\subsection{Gabor analysis of $\tau$-pseudodifferential operators}

For $\tau \in[0,1], f, g \in L^{2}\left(\mathbb{R}^{d}\right)$, the (cross-) $\tau$-Wigner distribution is defined by

$$
W_{\tau}(f, g)(x, \omega)=\int_{\mathbb{R}^{d}} e^{-2 \pi i y \omega} f(x+\tau y) \overline{g(x-(1-\tau) y)} d y, \quad x, \omega \in \mathbb{R}^{d} .
$$

It can be used to define the $\tau$-pseudodifferential operator with symbol $\sigma$ via the formula

$$
\left\langle\mathrm{Op}_{\tau}(\sigma) f, g\right\rangle=\left\langle\sigma, W_{\tau}(g, f)\right\rangle, \quad f, g \in \mathcal{S}\left(\mathbb{R}^{d}\right) .
$$

For $\tau=1 / 2$ we recapture the Weyl operator. We want to consider $\tau$-pseudodifferential operators with symbols $\sigma$ in the Hörmander class $S_{0,0}^{m}, m \in \mathbb{R}$, consisting of functions $\sigma \in \mathcal{C}^{\infty}\left(\mathbb{R}^{2 d}\right)$ such that, for every $\alpha \in \mathbb{N}^{2 d}$,

$$
\left|\partial^{\alpha} \sigma(z)\right| \leq C_{\alpha}\langle z\rangle^{m}, \quad z \in \mathbb{R}^{2 d}
$$

The related semi-norms are denote by

$$
|\sigma|_{N, m}:=\sup _{|\alpha| \leq N}\left|\partial^{\alpha} \sigma(z)\right|\langle z\rangle^{-m}
$$

Fix $g \in \mathcal{S}\left(\mathbb{R}^{d}\right) \backslash\{0\}$. We define the Gabor matrix of a linear continuous operator $T$ from $\mathcal{S}\left(\mathbb{R}^{d}\right)$ to $\mathcal{S}^{\prime}\left(\mathbb{R}^{d}\right)$, the mapping from $\mathbb{R}^{2 d} \times \mathbb{R}^{2 d}$ into $\mathbb{C}$,

$$
(z, y) \mapsto\langle T \pi(z) g, \pi(y) g\rangle, \quad z, y \in \mathbb{R}^{2 d} .
$$

This is a slightly abuse of notation, since originally Gabor matrices we defined for time-frequency shifts $\pi(\lambda)$, with $\lambda$ varying in a lattice $\Lambda \subset \mathbb{R}^{2 d}$. We observe that the almost diagonalization of Gabor matrices of pseudodifferential operators with symbols in the modulation space $M^{\infty, 1}\left(\mathbb{R}^{2 d}\right)$ treated in [17] (and in many subsequent papers on the topic) are valid in both the continuous and discrete case. So we adopt this terminology in the continuous framework. 
For $m=0$ we are reduced to the Hörmander class $S_{0,0}^{0}$, whose Gabor matrix characterization for Weyl operators was shown in [18, Theorem 6.1], see also [23]. Even though $m=0$ is our case of interest, for our goal we need to control such matrix by the semi-norms of $S_{0,0}^{0}$. Moreover, for further references, we shall formulate our result in the case of $\tau$-pseudodifferential operators having symbols in the more general class $S_{0,0}^{m}, m \in \mathbb{R}$.

We are going to use the following result for $\tau$-pseudodifferential operators [11, Lemma 4.1].

Lemma 2.1 Fix a non-zero window $g \in \mathcal{S}\left(\mathbb{R}^{d}\right)$ and set $\Phi_{\tau}=W_{\tau}(g, g)$ for $\tau \in[0,1]$. Then, for $\sigma \in \mathcal{S}^{\prime}\left(\mathbb{R}^{2 d}\right)$,

$$
\left|\left\langle\mathrm{Op}_{\tau}(\sigma) \pi(z) g, \pi(y) g\right\rangle\right|=\left|V_{\Phi_{\tau}} \sigma\left(\mathcal{T}_{\tau}(z, y), J(y-z)\right)\right| .
$$

where $z=\left(z_{1}, z_{2}\right), y=\left(y_{1}, y_{2}\right)$ and $\mathcal{T}_{\tau}$ and $J$ are defined as follows:

$$
\mathcal{T}_{\tau}(z, y)=\left((1-\tau) z_{1}+\tau y_{1}, \tau z_{2}+(1-\tau) y_{2}\right), \quad J(z)=\left(z_{2},-z_{1}\right) .
$$

The Gabor matrix for a $\tau$-pseudodifferential operator $\operatorname{Op}_{\tau}(\sigma)$ with symbol $\sigma \in S_{0,0}^{m}$ enjoys the following decay.

Theorem 2.4 Fix $g \in \mathcal{S}\left(\mathbb{R}^{d}\right) \backslash\{0\}, m \in \mathbb{R}, \tau \in[0,1]$. Consider a $\tau$-pseudodifferential operator $\mathrm{Op}_{\tau}(\sigma)$ with symbol $\sigma \in S_{0,0}^{m}$. Then for every $N \in \mathbb{N}$ there exists $C=$ $C(N)>0$ such that

$$
\left|\left\langle\mathrm{Op}_{\tau}(\sigma) \pi(z) g, \pi(y) g\right\rangle\right| \leq C|\sigma|_{2 N, m} \frac{\left\langle\mathcal{T}_{\tau}(z, y)\right\rangle^{m}}{\langle y-z\rangle^{2 N}}, \quad z, y \in \mathbb{R}^{2 d},
$$

where the semi-norms $|\cdot|_{N, m}$ are defined in (19).

Proof Using the representation in (21) and

$$
\left(1-\Delta_{\lambda}\right)^{N} e^{-2 \pi i \lambda J(y-z)}=\langle 2 \pi(y-z)\rangle^{2 N} e^{-2 \pi i \lambda J(y-z)}
$$

we can write

$$
\begin{aligned}
\left|\left\langle\mathrm{Op}_{\tau}(\sigma) \pi(z) g, \pi(y) g\right\rangle\right| \leq & C \frac{1}{\langle 2 \pi(y-z)\rangle^{2 N}} \\
& \times\left|\int_{\mathbb{R}^{2 d}} e^{-2 \pi i \lambda J(y-z)}\left(1-\Delta_{\lambda}\right)^{N}\left[\bar{\sigma}(\lambda) T_{\mathcal{T}_{\tau}(z, y)} \Phi_{\tau}\right] d \lambda\right|
\end{aligned}
$$

(observe that the above integral is absolutely convergent since $\Phi_{\tau} \in \mathcal{S}\left(\mathbb{R}^{2 d}\right)$ ). Now we estimate

$$
\begin{aligned}
\left(1-\Delta_{\lambda}\right)^{N}\left[\bar{\sigma}(\lambda) T_{\mathcal{T}_{\tau}(z, y)} \Phi_{\tau}\right] & \left.\leq \sum_{|\alpha|+|\beta| \leq 2 N}\left|C_{\alpha, \beta}\right|\left|\partial^{\alpha} \sigma(\lambda)\right| \mid \partial^{\beta} \Phi_{\tau}\left(\lambda-\mathcal{T}_{\tau}(z, y)\right)\right) \mid \\
& \leq C_{N}|\sigma|_{2 N, m}\langle\lambda\rangle^{m}\left\langle\lambda-\mathcal{T}_{\tau}(z, y)\right\rangle^{-s}
\end{aligned}
$$


for every $s \geq 0$ since $\Phi_{\tau} \in \mathcal{S}\left(\mathbb{R}^{2 d}\right)$. Choose $s=|m|+2 d+1$. Then the submultiplicativity of $\langle\cdot\rangle^{|m|}$ allows us to control from above the right-hand side of the last inequality by

$$
C_{N}|\sigma|_{2 N, m}\left\langle\mathcal{T}_{\tau}(z, y)\right\rangle^{m}\left\langle\lambda-\mathcal{T}_{\tau}(z, y)\right\rangle^{-(2 d+1)}
$$

Hence, for every $N \in \mathbb{N}$ we can find $C(N)>0$ such that (22) is satisfied. This concludes the proof.

For fixed $\tau \in(0,1)$ we observe that $\left\langle\mathcal{T}_{\tau}(z, y)\right\rangle \asymp\langle z+y\rangle$, hence the matrix decay can be controlled by a function which does not depend on the $\tau$-quantization. Namely,

Corollary 2.5 Fix $g \in \mathcal{S}\left(\mathbb{R}^{d}\right) \backslash\{0\}, m \in \mathbb{R}, \tau \in(0,1)$. Consider a $\tau$-pseudodifferential operator $\mathrm{Op}_{\tau}(\sigma)$ with symbol $\sigma \in S_{0,0}^{m}$. Then, for every $N \in \mathbb{N}$, there exists $C=$ $C(\tau, N)>0$ such that

$$
\left|\left\langle\mathrm{Op}_{\tau}(\sigma) \pi(z) g, \pi(y) g\right\rangle\right| \leq C|\sigma|_{2 N, m} \frac{\langle z+y\rangle^{m}}{\langle y-z\rangle^{2 N}}, \quad z, y \in \mathbb{R}^{2 d} .
$$

Remark 2.2 We conjecture that pseudodifferential operators in the Hörmander class $S_{0,0}^{m}, m \in \mathbb{R}$, can be characterized via the Gabor matrix in (22), extending the case $m=0$ already shown in [18]. To be precise, we allow to write

$$
S_{0,0}^{m}=\bigcap_{s \geq 0} M_{\langle\cdot\rangle^{m} \otimes\langle\cdot\rangle^{s}}^{\infty}=\bigcap_{s \geq 0} M_{\langle\cdot\rangle^{m} \otimes\langle\cdot\rangle^{s}}^{\infty, 1}
$$

Studying the Gabor matrix decay for $M_{\langle\cdot\rangle^{m}}^{\infty} \otimes\langle\cdot\rangle^{s}$ and following the pattern of the proofs as in the paper [18] one should get the result easily. Since this subject is outside the scope of the paper, we will write the details in a separate work.

\section{Local well-posedness in modulation spaces}

For $m=0$ the semi-norms on $\Gamma_{1}^{0}$ are exactly the ones in (15). Observe that

$$
\Gamma_{1}^{m} \hookrightarrow S_{0,0}^{m}
$$

(the inclusion is continuous).

The results in the previous yields the boundedness of the Weyl operator $e^{-t H^{\beta}}$ on modulation spaces.

Theorem 3.1 Consider $1 \leq p, q \leq \infty, 0<\beta \leq 1, w \in \mathcal{M}_{v}$. Then for every $T>0$ there exists $C=C(T)>0$ such that

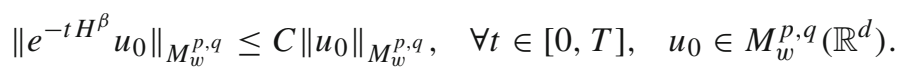


Proof Consider $u_{0} \in M_{w}^{p, q}\left(\mathbb{R}^{d}\right)$. Fix $g \in \mathcal{S}\left(\mathbb{R}^{d}\right) \backslash\{0\}$ such that $\|g\|_{2}=1$. Then using the inversion formula for $u_{0}$ in (8) we can write

$$
\begin{aligned}
\left|V_{g}\left(e^{-t H^{\beta}} u_{0}\right)(y) w(y)\right| & =\left|\int_{\mathbb{R}^{2 d}}\left\langle e^{-t H^{\beta}} \pi(z) g, \pi(y) g\right\rangle V_{g} u_{0}(z) d z\right| \\
& \leq \int_{\mathbb{R}^{2 d}} w(y)\left|\left\langle e^{-t H^{\beta}} \pi(z) g, \pi(y) g\right\rangle\right|\left|V_{g} u_{0}(z)\right| d z
\end{aligned}
$$

In the previous section we showed that $e^{-t H^{\beta}}$ is a Weyl operator with symbol $b(t, \cdot)$ in $\Gamma_{1}^{0}$ with semi-norms uniformly bounded w.r.t. $t \in[0, T]$. The continuous embedding in (24) and Theorem 2.4 let us write

$$
\left|\left\langle e^{-t H^{\beta}} \pi(z) g, \pi(y) g\right\rangle\right| \leq C \frac{1}{\langle y-z\rangle^{2 N}} .
$$

Since $w(y) \lesssim v(y-z) w(z)$, and $v(z) \lesssim\langle z\rangle^{s}$ for some $s>0$, we can write

$$
\begin{aligned}
\left|V_{g}\left(e^{-t H^{\beta}} u_{0}\right)(y) w(y)\right| & \leq C \int_{\mathbb{R}^{2 d}}\langle y-z\rangle^{s} w(z) \mid V_{g} u_{0}(z) \frac{1}{\langle y-z\rangle^{2 N}} d z \\
& \leq C\left[\frac{1}{\langle\cdot\rangle^{2 N-s}} *\left(\left|V_{g} u_{0}\right| w\right)\right](y)
\end{aligned}
$$

Choosing $N$ such that $2 N-s>2 d+1$ and using the convolution relations $L^{1} * L^{p, q} \hookrightarrow$ $L^{p, q}$ we obtain the claim.

Choosing $p=q=2$ and recalling that, for $w(x, \xi)=\langle\xi\rangle^{s}, M_{w}^{2}\left(\mathbb{R}^{d}\right)=H^{s}\left(\mathbb{R}^{d}\right)$ (Sobolev spaces), whereas for $w(z)=\langle z\rangle^{s}, z \in \mathbb{R}^{2 d}, M_{w}^{2}\left(\mathbb{R}^{d}\right)=\mathcal{Q}_{s}$ (Shubin-Sobolev spaces), cf., e.g., [9, Chapter 2], we obtain boundedness results also for these classical spaces.

Corollary 3.2 Consider $0<\beta \leq 1, s \in \mathbb{R}$. For any fixed $T>0$ there exists $C=$ $C(T)>0$ such that

$$
\left\|e^{-t H^{\beta}} u_{0}\right\|_{H^{s}} \leq C\left\|u_{0}\right\|_{H^{s}}, \quad \forall t \in[0, T], \quad u_{0} \in H^{s}\left(\mathbb{R}^{d}\right) .
$$

The same result holds by replacing the Sobolev space $H^{s}$ with the Shubin-Sobolev space $\mathcal{Q}_{s}$.

As already done in [12], in order to show the local existence of the solution we will make use of the following variant of the contraction mapping theorem (cf., e.g., [26, Proposition 1.38]).

Proposition 3.3 Let $\mathcal{N}$ and $\mathcal{T}$ be two Banach spaces. Consider a linear operator $\mathcal{B}: \mathcal{N} \rightarrow \mathcal{T}$ such that

$$
\|\mathcal{B} f\|_{\mathcal{T}} \leq C_{0}\|f\|_{\mathcal{N}}, \quad \forall f \in \mathcal{N}
$$


for some $C_{0}>0$, and suppose to have a nonlinear operator $F: \mathcal{T} \rightarrow \mathcal{N}$ with $F(0)=0$ and Lipschitz bounds

$$
\|F(u)-F(v)\|_{\mathcal{N}} \leq \frac{1}{2 C_{0}}\|u-v\|_{\mathcal{T}},
$$

for all $u, v$ in the ball $B_{\mu}:=\left\{u \in \mathcal{T}:\|u\|_{\mathcal{T}} \leq \mu\right\}$, for some $\mu>0$. Then, for all $u_{\text {lin }} \in B_{\mu / 2}$ there exists a unique solution $u \in B_{\mu}$ to the equation $u=u_{\text {lin }}+\mathcal{B} F(u)$, with the map $u_{\text {lin }} \mapsto u$ Lipschitz continuous with constant at most 2.

Proof of Theorem 1.1 We apply Theorem 3.1 with $T=1, q=1$ and $w(x, \xi)=\langle\xi\rangle^{s}$. For every $1 \leq p<\infty$, the operator $K_{\beta}(t)$ in (3) is a bounded operator on $M_{S}^{p, 1}\left(\mathbb{R}^{d}\right)$, and there exists a $C>0$ such that

$$
\left\|K_{\beta}(t) u_{0}\right\|_{M_{s}^{p, 1}} \leq C\left\|u_{0}\right\|_{M_{s}^{p, 1}}, \quad t \in[0,1]
$$

Notice that such result provides the uniformity of the constant $\mathrm{C}$, when t varies in $[0,1]$. Now the result follows by Proposition 3.3, with $\mathcal{T}=\mathcal{N}=C^{0}\left([0, T] ; M_{s}^{p, 1}\right)$, the linear operator $\mathcal{B}$ in (3), where $0<T \leq 1$ will be chosen later on. Here $u_{\text {lin }}:=K_{\beta}(t) u_{0}$ is in the ball $B_{\mu / 2} \subset \mathcal{T}$ by (29), if $\mu$ is sufficiently large, depending on the radius $R$ in $M_{s}^{p, 1}\left(\mathbb{R}^{d}\right)$ in the assumptions. Using Minkowski's integral inequality and (29), we obtain (27). Namely,

$$
\|\mathcal{B} u\|_{M_{s}^{p, 1}} \leq T C\|u\|_{M_{s}^{p, 1}}
$$

The proof of Condition (28) can be found in [8, proof of Theorem 4.1]. Hence, by choosing $T$ small enough we prove the existence, and also the uniqueness among the solution in $\mathcal{T}$ with norm $O(R)$ (with $R$ being the radius of the ball $B_{R}$, centred in 0 , in $\left.M_{S}^{p, 1}\left(\mathbb{R}^{d}\right)\right)$. Standard continuity arguments allow to eliminate the last constraint (see, e.g., [26, Proposition 3.8]). For $p=\infty$, by repeating the argument above, one can obtain well-posedness when the initial datum is in

$$
\mathcal{M}_{s}^{\infty, 1}\left(\mathbb{R}^{d}\right):=\overline{\mathcal{S}}^{M_{s}^{\infty, 1}}\left(\mathbb{R}^{d}\right)
$$

Observe that similar results were obtained in [21, Theorem 1.1].

We conclude this note by addressing the reader to open problems in this field.

First, it is still not clear whether better results can be obtained when considering the nonlinearity

$$
F(u)=F_{k}(u)=\lambda|u|^{2 k} u=\lambda u^{k+1} \bar{u}^{k}, \quad \lambda \in \mathbb{C}, k \in \mathbb{N} .
$$

In fact, this was the case for the wave and vibrating place equation, cf. [8,12], where more general modulation spaces were considered. 
Moreover, another open question is the well-posedness of the Cauchy problem (1) with initial datum $u_{0} \in M_{s}^{p, q}\left(\mathbb{R}^{d}\right), 0<p \leq \infty, 0<q \leq 1$. We conjecture that the result holds true as well, but the techniques employed so far do not apply in this case.

Acknowledgements The author would like to thank Professors Fabio Nicola and Luigi Rodino for fruitful conversations and comments.

Funding Open access funding provided by Università degli Studi di Torino.

Open Access This article is licensed under a Creative Commons Attribution 4.0 International License, which permits use, sharing, adaptation, distribution and reproduction in any medium or format, as long as you give appropriate credit to the original author(s) and the source, provide a link to the Creative Commons licence, and indicate if changes were made. The images or other third party material in this article are included in the article's Creative Commons licence, unless indicated otherwise in a credit line to the material. If material is not included in the article's Creative Commons licence and your intended use is not permitted by statutory regulation or exceeds the permitted use, you will need to obtain permission directly from the copyright holder. To view a copy of this licence, visit http://creativecommons.org/licenses/by/4.0/.

\section{References}

1. Bényi, A., Gröchenig, K., Okoudjou, K.A., Rogers, L.G.: Unimodular Fourier multipliers for modulation spaces. J. Funct. Anal. 246(2), 366-384 (2007)

2. Bényi, A., Okoudjou, K.A.: Local well-posedness of nonlinear dispersive equations on modulation spaces. Bull. Lond. Math. Soc. 41(3), 549-558 (2009)

3. Bhimani, D.G.: The Nonlinear heat equations with fractional Laplacian and harmonic oscillator in modulation spaces. arXiv:1911.01844

4. Bhimani, D.G., Balhara, R., Thangavelu, S.: Hermite multipliers on modulation spaces. Analysis and partial differential equations: perspectives from developing countries, 42-64, Springer Proc. Math. Stat., 275, Springer, Cham (2019)

5. Bhimani, D.G., Manna, R., Nicola, F., Thangavelu, S., Trapasso, S.I.: Optimal estimates for the Hermite semigroup on modulation spaces, in preparation

6. Catană, V.: The heat equation for the generalized Hermite and the generalized Landau operators. Integr. Equ. Oper. Theory 66(1), 41-52 (2010)

7. Cordero, E., de Gosson, M., Nicola, F.: Born-Jordan pseudo-differential operators with symbols in the shubin classes. Trans. Am. Math. Soc. 4, 94-109 (2017)

8. Cordero, E., Nicola, F.: Remarks on Fourier multipliers and applications to the wave equation. J. Math. Anal. Appl. 353(2), 583-591 (2009)

9. Cordero, E., Rodino, L.: Time-frequency Analysis of Operators, De Gruyter, to appear

10. Cordero, E., Nicola, F., Rodino, L.: Gabor Representations of evolution operators. Trans. Am. Math. Soc. 367(11), 7639-7663 (2015)

11. Cordero, E., Nicola, F., Trapasso, S.I.: Almost diagonalization of $\tau$-pseudodifferential operators with symbols in Wiener amalgam and modulation spaces. J. Fourier Anal. Appl. 25(4), 1927-1957 (2019)

12. Cordero, E., Zucco, D.: The Cauchy problem for the vibrating plate equation in modulation spaces. J. Pseudo-Differ. Op. Appl. 2, 343-354 (2011)

13. de Gosson, M.: Symplectic methods in harmonic analysis and in mathematical physics, Birkhäuser, (2011)

14. Feichtinger, H.G.: Modulation spaces on locally compact abelian groups, Technical Report, University Vienna, 1983. and also in Wavelets and Their Applications, M. Krishna, R. Radha, S. Thangavelu, editors, Allied Publishers, 99-140, 2003

15. Galperin, Y.V., Samarah, S.: Time-frequency analysis on modulation spaces $M_{m}^{p, q}, 0<p, q \leq \infty$. Appl. Comput. Harmon. Anal. 16(1), 1-18 (2004)

16. Gröchenig, K.: Foundation of Time-Frequency Analysis. Birkhäuser, Boston MA (2001)

17. Time-frequency analysis of Sjöstrand's class. Rev. Mat. Iberoam. 22(2), 703-724 (2006)

18. Gröchenig, K., Rzeszotnik, Z.: Banach algebras of pseudodifferential operators and their almost diagonalization. Ann. Inst. Fourier. 58(7), 2279-2314 (2008) 
19. Guo, W., Chen, J., Fan, D., Zhao, G.: Characterizations of some properties on weighted modulation and Wiener amalgam spaces. Michigan Math. J. 68(3), 451-482 (2019)

20. Helffer, B.: Théorie spectrale pour des opérateurs globalement elliptiques. Astérisque, 112. Société Mathématique de France, Paris, 1984

21. Nicola, F.: Phase space analysis of semilinear parabolic equations. J. Funct. Anal. 267(3), 727-743 (2014)

22. Nicola, F., Rodino, L.: Global pseudo-differential calculus on Euclidean spaces. Pseudo-Differential Operators. Theory and Applications, 4. Birkhäuser Verlag, Basel, 2010

23. Rochberg, R., Tachizawa, K.: Pseudodifferential operators, Gabor frames, and local trigonometric bases, in Gabor Analysis and Algorithms, pp. 171-192. Birkhäuser Boston, Boston, MA, Appl. Numer. Harmon. Anal. (1998)

24. Shubin, M.A.: Pseudodifferential Operators and Spectral Theory. Springer Series in Soviet Mathematics. Springer, Berlin (1987)

25. Thangavelu, S.: Lectures on Hermite and Laguerre Expansions. Mathematical Notes-Princeton University Press, (1993)

26. Tao, T.: Nonlinear Dispersive Equations: Local and Global Analysis. In: CBMS Regional Conference Series in Mathematics, American Mathematical Society (2006)

27. Wang, B., Chunyan, H.: Frequency-uniform decomposition method for the generalized BO, KdV and NLS equations. J. Diff. Equ. 239, 213-250 (2007)

28. Wang, B., Hudzik, H.: The global Cauchy problem for the NLS and NLKG with small rough data. J. Differ. Equ. 232, 36-73 (2007)

29. Wang, B., Zhao, L., Guo, B.: Isometric decomposition operators, function spaces $E_{p, q}^{\lambda}$ and applications to nonlinear evolution equations. J. Funct. Anal. 233(1), 1-39 (2006)

30. Wang, B., Huo, Z., Hao, C., Guo, Z.: Harmonic analysis method for nonlinear evolution equations. I. World Scientific Publishing Co. Pte. Ltd., Hackensack, NJ, (2011)

31. Wong, M.W.: The heat equation for the Hermite operator on the Heisenberg group. Hokkaido Math. J. 34(2), 393-404 (2005)

32. Wong, M.W.: Weyl transforms, the heat Kernel and Green function of a degenerate elliptic operator. Ann. Glob. Anal. Geom. 28, 271-283 (2005)

Publisher's Note Springer Nature remains neutral with regard to jurisdictional claims in published maps and institutional affiliations. 